\title{
Enhanced binding of antibodies generated during chronic HIV infection to mucus component MUC16
}

\author{
BM Gunn ${ }^{1,13}$, JR Schneider ${ }^{2,13}$, M Shansab ${ }^{1,3,13}$, AR Bastian $^{4}$, KM Fahrbach ${ }^{2}$, AD SmithIV $^{5}$, AE Mahan $^{1}$, \\ MM Karim ${ }^{1}$, AF Licht ${ }^{1}$, I Zvonar ${ }^{1}$, J Tedesco ${ }^{1}$, MR Anderson ${ }^{2}$, A Chapel ${ }^{1,6}$, TJ Suscovich ${ }^{1}$, DC Malaspina ${ }^{4}$, \\ H Streeck $^{7}$, BD Walker ${ }^{1}$, A Kim ${ }^{8}$, G Lauer ${ }^{8}$, M Altfeld ${ }^{1,6}$, S Pillai ${ }^{1,8}$, I Szleifer ${ }^{4,9,10}$, NL Kelleher ${ }^{5,9,10,11}$, PF \\ Kiser $^{4,9,12}$, TJ Hope ${ }^{2,4,9,12}$ and G Alter ${ }^{1}$
}

Transmission of HIV across mucosal barriers accounts for the majority of HIV infections worldwide. Thus, efforts aimed at enhancing protective immunity at these sites are a top priority, including increasing virus-specific antibodies (Abs) and antiviral activity at mucosal sites. Mucin proteins, including the largest cell-associated mucin, mucin 16 (MUC16), help form mucus to provide a physical barrier to incoming pathogens. Here, we describe a natural interaction between Abs and MUC16 that is enhanced in specific disease settings such as chronic HIV infection. Binding to MUC16 was independent of IgG subclass, but strongly associated with shorter Ab glycan profiles, with agalactosylated (G0) Abs demonstrating the highest binding to MUC16. Binding of Abs to epithelial cells was diminished following MUC16 knockdown, and the MUC16 N-linked glycans were critical for binding. Further, agalactosylated VRC01 captured HIV more efficiently in MUC16. These data point to a novel opportunity to enrich Abs at mucosal sites by targeting Abs to MUC16 through changes in Fc glycosylation, potentially blocking viral movement and sequestering the virus far from the epithelial border. Thus, next-generation vaccines or monoclonal therapeutics may enhance protective immunity by tuning Ab glycosylation to promote the enrichment of Abs at mucosal barriers.

\section{INTRODUCTION}

Sexual transmission of HIV across mucosal barriers accounts for the majority of new HIV infections each year. Women are at particular risk of infection, with young women twice as likely as young men to be infected with HIV via heterosexual transmission. ${ }^{1,2}$ Although an effective HIV vaccine remains elusive, enhancing protection at mucosal sites is key to provide protective immunity in next-generation vaccine strategies. Passive transfer of neutralizing antibodies (Abs) against HIV can provide protection against mucosal challenge, ${ }^{3,4}$ and beyond neutralization, Fc-mediated Ab effector functions have been implicated in protection against HIV., Recently, targeting of broadly neutralizing Abs to mucosal compartments resulted in increased protection of non-human primates from mucosal challenge, ${ }^{7}$ suggesting that strategies aimed at increasing the concentration of virus-specific Abs at mucosal sites may provide enhanced protection from infection. However, as vaccination cannot induce mutations in the $\mathrm{Fc}$, identifying natural $\mathrm{Ab}$ modifications that increase $\mathrm{Ab}$ concentration at mucosal sites represents a novel opportunity to enhance immunity against HIV and other mucosal pathogens.

All mucosal surfaces are lined with a thick layer of mucus that provides a protective physical barrier for the underlying epithelium by trapping pathogens and microbes. Anti-microbial peptides, immune proteins, and Abs are present within mucus, and can be bound to a lattice of heavily glycosylated

\footnotetext{
${ }^{1}$ Ragon Institute of MGH, MIT, and Harvard, Cambridge, Massachusetts, USA. ${ }^{2}$ Department of Cell and Molecular Biology, Feinberg School of Medicine, Northwestern University, Chicago, Illinois, USA. ${ }^{3}$ Department of Microbiology and Immunology, University of California San Francisco, San Francisco, California, USA. ${ }^{4}$ Department of Biomedical Engineering, Northwestern University, Evanston, Illinois, USA. ${ }^{5}$ Proteomics Center of Excellence, Northwestern University, Evanston, Illinois, USA. ${ }^{6}$ Department of Virus Immunology, Heinrich-Pette-Institut, Hamburg, Germany. ${ }^{7}$ Military HIV Research Program, Bethesda, Maryland, USA. ${ }^{8}$ Masschusetts General Hospital, Boston, Massachusetts, USA. ${ }^{9}$ Chemistry of Life Processes Institute, Northwestern University, Evanston, Illinois, USA. ${ }^{10}$ Department of Chemistry, Northwestern University, Evanston, Illinois, USA. ${ }^{11}$ Department of Molecular Biosciences, Northwestern University, Evanston, Illinois, USA and ${ }^{12}$ Department of Obstetrics and Gynecology, Northwestern University, Chicago, Illinois, USA. Correspondence: TJ Hope or G Alter, (thope@northwestern.edu) or (galter@mgh.harvard.edu)

${ }^{13}$ These authors contributed equally to this work. 
mucin proteins that line the membranes. ${ }^{8}$ The specific mechanisms by which these immune proteins are bound in mucus are not fully understood but may hold the key to vaccine or therapeutic strategies aimed at enriching antiviral Abs along these vulnerable tissues. Cell-associated mucin proteins including the largest mucin, mucin 16 (MUC16), line the endocervix, endometrium, and fallopian tubes to provide an additional barrier for pathogens to overcome in order to reach the epithelium. ${ }^{9,10}$ Because the endocervix is lined by a single layer of columnar epithelial cells that is highly susceptible to infection by HIV, ${ }^{11}$ the mucin barrier provides an additional protective layer against infectious agents.

In this study, we aimed to determine whether Abs could be selectively enriched at mucosal barriers, ultimately identifying novel means to promote higher concentrations of HIV-specific Abs at these sites. Here, we identified an interaction between IgG and the mucin, MUC16, which is selectively enhanced in chronic $\mathrm{HIV}^{+}$subjects. Specifically, particular Fc glycosylation patterns, independent of $\mathrm{Ab}$ subclass, were associated with enhanced binding to MUC16, and manipulation of the glycan structure modulated MUC16 binding interactions, and subsequent capture of virus. Together, these data highlight a novel opportunity to promote HIV-specific Ab enrichment above mucosal membranes through altered Ab glycosylation that may immobilize incoming virus to provide enhanced protection from infection.

\section{RESULTS}

\section{Abs from HIV ${ }^{+}$patients preferentially bind to MUC16}

Previous studies demonstrate increased levels of IgG1 and IgG3 Abs in the cervicovaginal secretions (CVS) of $\mathrm{HIV}^{+}$compared with HIV-negative women. ${ }^{12}$ Although the increased amounts of IgG in CVS likely result from hypergammaglobulinemia associated with HIV infection, ${ }^{13}$ we reasoned that there might be specific interactions between $\mathrm{Abs}$ from $\mathrm{HIV}^{+}$individuals and mucus proteins that may allow them to retain high levels of Abs within mucus. Thus, to determine whether specific proteins in mucus bind differentially to Abs during HIV infection, we examined the capacity of Abs isolated from chronic $\mathrm{HIV}^{+}$ patients or healthy controls to bind to a number of proteins that associate with epithelial cells at mucosal membranes. Among the proteins found at these sites that may interact with Abs, ${ }^{14}$ no differences were observed in $\mathrm{Ab}$ binding to galectin proteins Gal-1, Gal-3, Gal-7, and Gal-9 (Figure 1a). Next, we probed the capacity of Abs to interact with some of the most abundant proteins at the mucosal barrier in the female reproductive tract, the membrane-associated mucin proteins, MUC1 and MUC16. Although Abs were able to bind to recombinant fragments of both mucins, limited differences were observed between the groups in binding to MUC1 (Figure 1b, left). In contrast, Abs purified from subjects with chronic HIV infection exhibited significantly enhanced binding to MUC16 (Figure 1b, right).

As the endocervix is vulnerable to HIV infection, we next determined whether both Abs and MUC16 are present in the same region of the reproductive tract. Thus, an endocervical explant from a healthy donor was stained for the presence of IgG and MUC16 and visualized by fluorescence microscopy. Consistent with previous studies demonstrating the presence of MUC16 lining the endocervix, ${ }^{8,15}$ MUC16 staining was observed on the apical side of the columnar epithelium (Figure 1c). The staining of the MUC16 at the epithelial border across the entire tissue section highlights the ideal localization of MUC16-mediated Ab enrichment to protect the underlying epithelium. Dispersed IgG staining was observed throughout the tissue section, consistent with prior studies demonstrating the presence of IgG within the reproductive tract. ${ }^{16,17}$ Interestingly, an accumulation of IgG was observed at the apical side of the columnar epithelial cells, overlapping with surfaces occupied by MUC16. Thus, interactions between Abs and MUC16 have the potential to concentrate Abs within the glycocalyx covering the luminal surface of the endocervix, enhancing mucosal barrier function.

Native MUC16 within tissues is much larger and likely glycosylated differently from recombinant MUC16, thus we next determined whether Abs from $\mathrm{HIV}^{+}$patients could also interact with naturally produced MUC16. Native MUC16 was purified from OVCAR3 cells and binding of Abs was evaluated. Consistent with our results using recombinant MUC16, $\mathrm{HIV}^{+}$ Abs demonstrated enhanced binding to native MUC16 compared with Abs from healthy controls (Figure 1d). To determine whether purified MUC16 was conformationally distinct on the cell surface, potentially abrogating Ab binding, Abs from $\mathrm{HIV}^{+}$ patients were incubated with either wild-type (WT) OVCAR3 cells, which naturally express MUC16, ${ }^{18}$ or MUC16-knockdown OVCAR3 cells. As shown in Figure 1e, Ab binding was detected on WT OVCAR3 cells, but reduced on MUC16-knockdown cells. Together, these data demonstrate that Abs can interact with native shed and cell-associated MUC16.

\section{Chronic HIV infection enhances the production of MUC16 binding Abs}

To begin to identify features of Abs that confer enhanced binding to MUC16, we sought to determine whether Abs with enhanced MUC16 binding are preferentially induced in subjects with different HIV-associated clinical disease outcomes, such as spontaneous control of HIV infection, previously associated with the induction of unique Ab Fc profiles. ${ }^{19}$ We compared Abs from HIV controllers and chronic HIV patients with and without antiretroviral treatment for binding to MUC16. Interestingly, no differences were observed in Ab binding to MUC16 between the different HIV patient groups, suggesting that HIV infection alone, rather than viremia, induces an increase in Abs with MUC16 binding abilities (Figure 1f).

The $\mathrm{Ab}$ response during HIV infection is dynamic, and Abs generated during acute infection differ from those produced during chronic infection with regard to subclass, antiviral function, and epitope specificity. ${ }^{13,20}$ Thus to determine at which point during infection Abs gain an enhanced capacity to bind to MUC16, Abs from acutely HIV-infected patients ( $<1$ year post infection) was compared with Abs from chronically infected $\mathrm{HIV}^{+}$patients ( $>2$ years) and healthy controls 

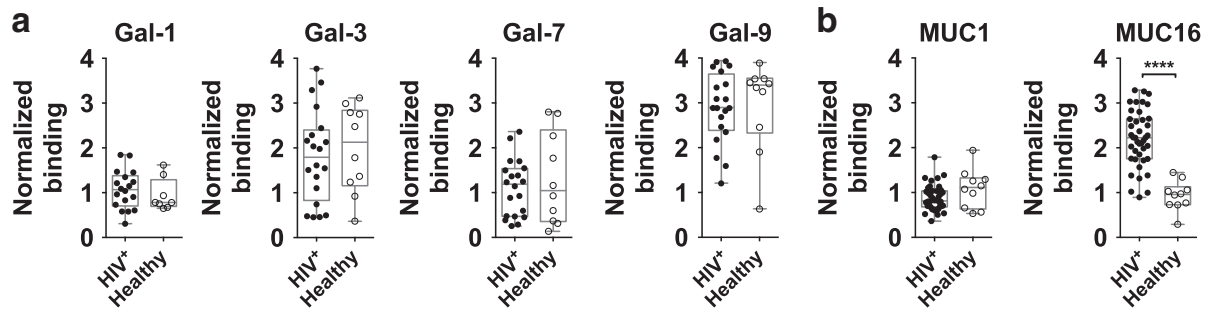

C
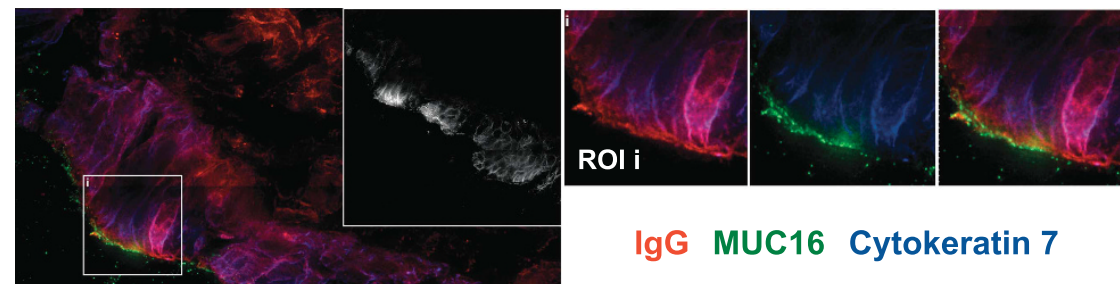

IgG MUC16 Cytokeratin 7
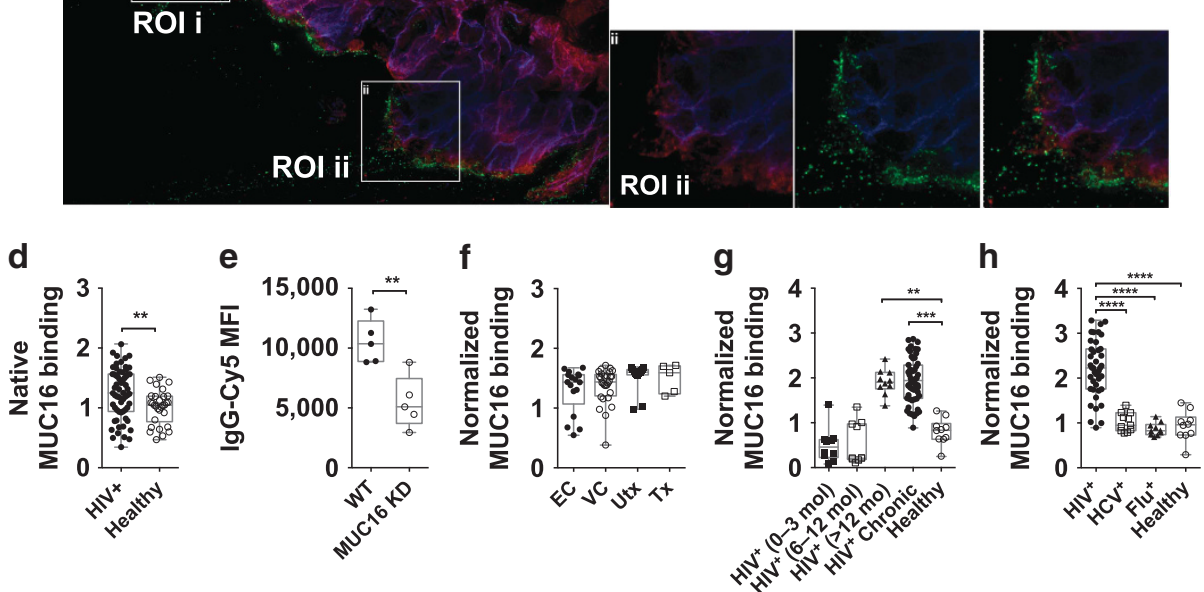

Figure 1 Antibodies (Abs) from HIV ${ }^{+}$patients preferentially bind to mucin 16 (MUC16). (a, b) Bulk IgG from HIV ${ }^{+}$patients or healthy subjects was assayed for binding to galectins (a) or mucins (b) by ELISA. (c) An endocervical explant from a donor was stained for MUC16 (FITC), cytokeratin 7 (blue; marks simple epithelium), and lgG (red) and imaged by fluorescence microscopy. The black and white inset shows overlap of IgG and MUC16. ROI i and ii show regions of cell-associated MUC16 (ROI i) as well as shed MUC16 (ROI ii). (d) Bulk IgG from HIV ${ }^{+}$patients or healthy subjects was assayed for binding to native MUC16 isolated from OVCAR3 cells by ELISA. (e) Bulk IgG from chronic $\mathrm{HIV}^{+}$patients was labeled with Cy5 and assayed for binding to either wild type (WT) or MUC16-knockdown OVCAR-3 cells by flow cytometry. (f) Bulk IgG from different HIV patient populations (elite (EC) or viremic controllers (VC); chronic progressor off ARV (UTx) or on ARV (Tx)) was assayed for MUC16 binding by ELISA. (g) Bulk IgG from acute (0-3 or 6-12 months) or chronic HIV infection (12 months; $>2$ years) or healthy subjects was assayed for binding to MUC16 by ELISA. (h) Bulk IgG from HIV ${ }^{+}$, HCV ${ }^{+}$, influenza patients or healthy subjects was assayed for binding to MUC16 by ELISA. ${ }^{\star \star} P<0.01,{ }^{\star \star \star} P<0.0005,{ }^{* \star \star *} P<0.0001$ by Mann-Whitney analysis (two groups) or Kruskal-Wallis analysis (three or more groups). FITC, fluorescein isothiocyanate; ROI, region of interest.

for MUC16 binding. Although limited binding was observed for Abs from acute infection, Abs from HIV patients infected for at least 1 year had enhanced MUC16 binding (Figure 1g). As hypergammaglobulinemia arises within the first 3 months of infection, ${ }^{13}$ it is likely that qualitative changes in the $\mathrm{Ab}$, rather than the total amount of $A b$, must change over the first year of $\mathrm{HIV}$ infection resulting in the generation of Abs with enhanced MUC16 binding abilities.

To determine whether Abs generated in other viral infections also exhibit amplified binding to MUC16, we examined the capacity of Abs induced during acute influenza infection or chronic hepatitis $\mathrm{C}$ virus (HCV) infection to bind to MUC16. Enhanced MUC16 binding was not observed in subjects with acute influenza infection or chronic HCV infection (Figure 1h). Combined, these data demonstrate that there is an increase in the amounts of Ab with optimal capacity to bind to MUC16 induced in the context of chronic HIV infection.

\section{Fc-mediated binding to MUC16}

As Abs can be divided into two distinct functional domainsthe variable Fab domain involved in antigen binding and the constant $\mathrm{Fc}$ domain involved in directing effector function - we next aimed to define which domain interacts preferentially with MUC16. Enzymatic digestion of a polyclonal pool of IgG from chronic HIV patients (HIVIG) was performed with papain or IdeS to produce $\mathrm{Fab}$ or $\mathrm{F}(\mathrm{ab})_{2}$, respectively, and $\mathrm{Fc}$ domains. Overall, the cleaved Fc domains from HIVIG demonstrated preferential binding to MUC16 compared with Fab and/or $\mathrm{F}(\mathrm{ab})_{2}$, with the $\mathrm{Fc}$ domain mediating approximately $60 \%$ of whole IgG binding to MUC16 (Figure 2a). To confirm the role of the Fc domain in the Ab/MUC16 interaction, we used a pair of monoclonal Abs (mAbs), VRC01 and Rituximab (RTX), that exemplify the two extremes (low and high) of MUC16 binding, representing a consistent surrogate of polyclonal IgG to dissect $\mathrm{Ab}$ determinants of binding (Figure 2b). VRC01 and RTX were 

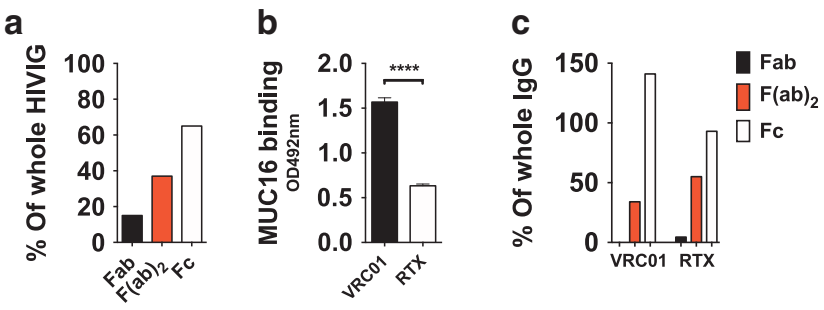

d

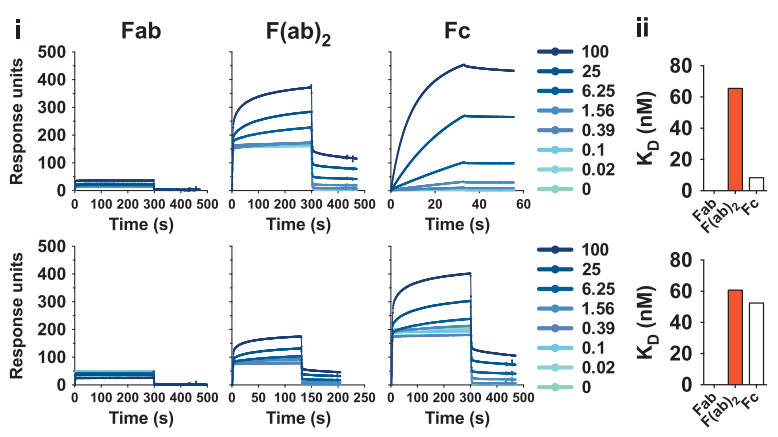

e
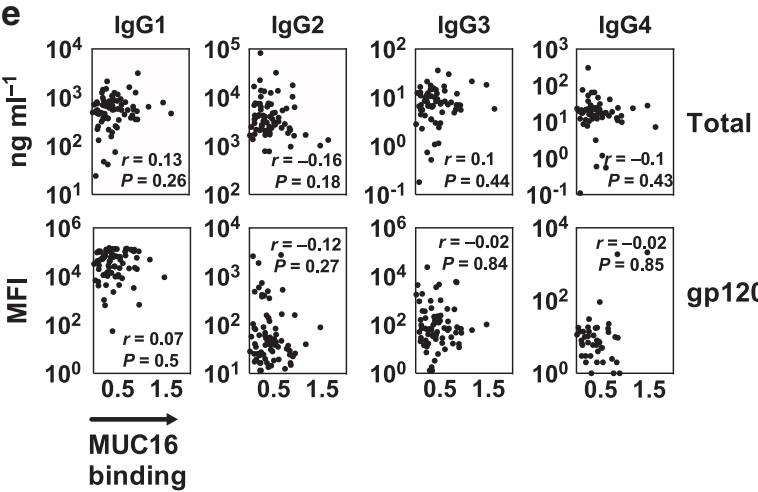
$0^{4}$\begin{tabular}{c|}
$r=-0.02$ \\
$P=0.85$
\end{tabular} gp120

Figure 2 FC-mediated binding to mucin 16 (MUC16). (a) HIVIG was cleaved with IdeS to generate $F(a b)_{2}$ fragments, or papain to generate intact Fc or Fab fragments, and assayed for MUC16 binding. The percent binding compared with whole lgG is shown. (b) VRC01 or Rituximab (RTX) was assayed for MUC16 binding by ELISA. (c) Fab, F(ab) 2 , or Fc fragments of VRC01 or RTX were assayed for MUC16 binding by ELISA. Percent binding of whole IgG is shown. (d) Fab, $F(a b)_{2}$, or Fc fragments of VRC01 or RTX were assayed for MUC16 binding by surface plasmon resonance (SPR). Raw SPR curves are shown in (i) and $K_{D}$ in (ii). (e) Total IgG subclass (top panel) or gp120-specific subclass titers (bottom panel) in IgG from HIV patients were determined by multiplex analysis. A Spearman correlation coefficient was used to determine correlations between MUC16 binding and Ab titers. ${ }^{\star \star \star \star} P<0.0001$ by unpaired $t$-test.

cleaved to produce $\mathrm{Fab}$ or $\mathrm{F}(\mathrm{ab})_{2}$ and $\mathrm{Fc}$ domains, and binding to MUC16 was assessed. The Fc domain bound MUC16 at approximately equal levels as whole $\mathrm{IgG}$, whereas the $\mathrm{F}(\mathrm{ab})_{2}$ domain bound at approximately half of the binding of whole IgG (Figure 2c). As we observed $\mathrm{F}(\mathrm{ab})_{2}$ binding, but not Fab binding to MUC16, it is possible that the increased binding is attributable to the detection of two Fabs in the $\mathrm{F}(\mathrm{ab})_{2}$ cleavage condition by the anti-Fab detection reagent. Thus, we performed surface plasmon resonance (SPR) to determine the binding affinity of Fc, Fab, and F(ab) ${ }_{2}$ of VRC01 and RTX to MUC16. Consistent with our results in the ELISA, the Fc portion of the VRC01 bound to MUC16 with approximately eightfold greater affinity than $\mathrm{F}(\mathrm{ab})_{2}$, whereas binding of the Fab portion was undetectable (Figure 2d). These data suggest that while some level of $\mathrm{F}(\mathrm{ab})_{2}$ binding to MUC16 can occur, a majority of binding is mediated via the Fc domain. In addition, both the $\mathrm{Fc}$ and $\mathrm{F}(\mathrm{ab})_{2}$ of RTX showed reduced binding affinity to MUC16 comparable to the level of VRC01 $\mathrm{F}(\mathrm{ab})_{2}$ binding, further supporting the hypothesis that the Fc domain of Abs mediates differential binding to MUC16.

\section{Ab subclass does not account for differential MUC16 binding}

As the Fc domain of Abs can be rapidly modulated during an immune response to drive differential $\mathrm{Ab}$ functionality via subclass selection and glycosylation, ${ }^{21}$ we next aimed to determine whether specific subclasses preferentially enhanced binding to MUC16. No relationship was observed between bulk subclass Ab titers or gp120-specific subclass titers and MUC16 binding (Figure 2e), pointing to a non-subclass mediated mechanism for enhanced MUC16 binding.

\section{Elevated levels of agalactosylated Abs correlate with enhanced MUC16 binding}

The Fc domain of IgG contains a glycosylation site at N297, ${ }^{22}$ and beyond subclass selection differences, Ab Fc glycosylation can be tuned during an immune response, aimed at modulating $\mathrm{Ab}$ interactions with $\mathrm{Fc}$ receptors and complement. ${ }^{23}$ Moreover, the Ab glycan varies widely with age, sex, and autoimmune diseases, ${ }^{24}$ and significant changes in IgG glycosylation have been observed in HIV infection, marked by the accumulation of high levels of agalactosylated Abs, ${ }^{19,25}$ also observed in active rheumatoid arthritis (RA) flares. ${ }^{26}$ Given the similarity in Fc glycosylation in HIV and RA, we hypothesized that Abs from RA patients also exhibit enhanced binding to MUC16. Indeed, Abs from RA patients bound to MUC16 at higher levels, similar to those from HIV patients, and trended toward enhanced binding to MUC16 compared with Abs from healthy controls (Figure 3a). As it is unlikely that Abs from RA patients and HIV patients share similar antigen-binding domains and subclass selection profiles, these data suggest that alterations in Fc glycosylation may have a role for the observed increase in MUC16 binding. Interestingly, Abs from influenza and $\mathrm{HCV}$ infection exhibit lower levels of agalactosylation (Supplementary Figure S1 online), supporting the hypothesis that differential Fc glycosylation may modulate enhanced MUC16 binding.

Ab glycan structures can be divided into classes based on the number of galactose molecules incorporated into the glycan, with G0 containing no galactose, G1 containing one galactose, and G2 containing two galactose molecules. ${ }^{22}$ As HIV-infected subjects and RA patients exhibit an enrichment of G0 Abs, the relationship between the abundance of G0, G1, and G2 structures and MUC16 binding was explored in a population of $\mathrm{HIV}^{+}$patients with variable Ab glycosylation following glycan analysis by capillary electrophoresis. ${ }^{27}$ A significant positive correlation was observed between MUC16 binding and G0 glycan levels across the entire population (Figure $3 \mathbf{b}$ ). Conversely, a significant negative correlation was observed 
a

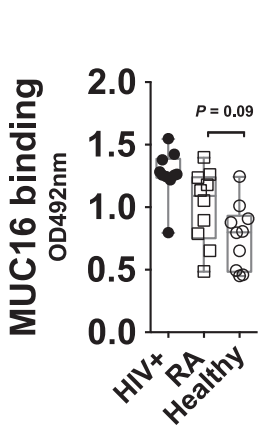

C

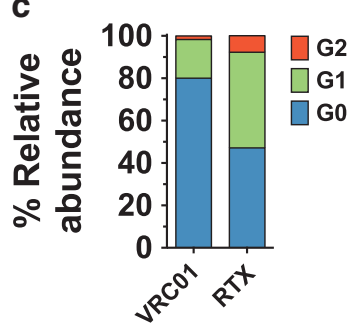

b

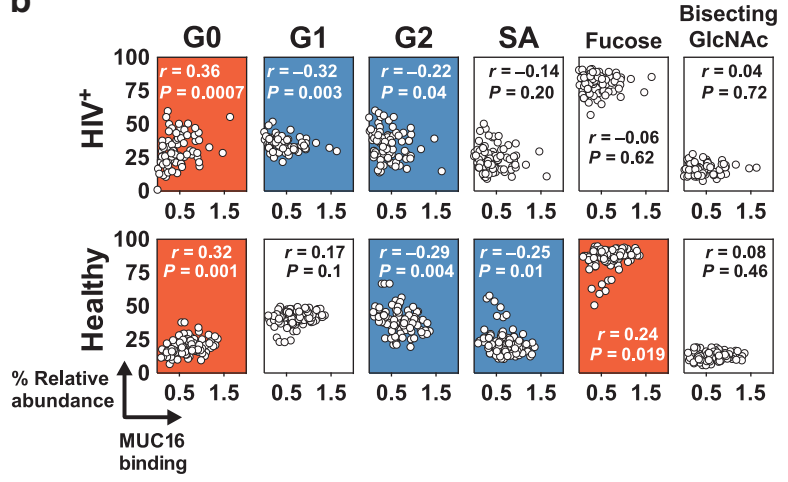

Figure 3 Elevated levels of agalactosylated antibodies (Abs) correlate with enhanced mucin 16 (MUC16) binding. (a) Bulk lgG from HIV ${ }^{+}$or rheumatoid arthritis (RA) patients or healthy subjects was assayed for binding to MUC16 by ELISA. (b) The relative abundance of glycan structures in bulk IgG from $\mathrm{HIV}^{+}$patients (top panel) or healthy subjects (bottom panel) was determined by capillary electrophoresis (CE). Spearman correlation coefficients indicate a significant positive (red), significant negative (blue), or no correlation (white) between relative glycan abundance and MUC16 binding. (c) The relative abundance of total G0, G1, and G2 glycan structures in VRC01 and Rituximab (RTX) was determined by mass spectrometry. (d) HIVIG was enriched for G0-Abs using ECL beads. Bound Abs were eluted and compared with unbound Abs for binding to MUC16 by ELISA. The data are expressed as percent binding to unenriched HIVIG Abs. (e) HIVIG was treated with or without $\beta$-galactosidase and assayed for MUC16 binding by ELISA. ${ }^{\star *} P<0.005$ by unpaired $t$-test.

with the relative abundance of G2 containing Abs, as G2 and G0 are inversely correlated. The same glycan/MUC16 binding relationships were confirmed in a replication cohort of acute and chronic HIV patients, influenza patients, HCV patients, RA patients, and healthy subjects (Supplementary Figure S2).

Although G0-containing Abs are enriched in HIV and RA patients compared with healthy subjects, G0-Abs are less abundant than G1 and G2 Abs in $\mathrm{HIV}^{+}$subjects (Supplementary Figure S3), suggesting that G0-Abs preferentially bind to MUC16. Moreover, although healthy patients show less binding to MUC16 relative to $\mathrm{HIV}^{+}$donors (Figure 1b), the abundance of G0-containing Abs also correlates with enhanced MUC16 binding in a healthy cohort (Figure 3b, bottom panel), consistent with a role for G0 glycosylation in the selective tuning of MUC16 binding, regardless of patient population. mAb glycosylation varies due to differences in the host expression cell line, ${ }^{28}$ and as VRC01 bound better than RTX to MUC16 (Figure 2b), we determined the relative percentage of G0, G1, and G2 glycan structures on these mAbs. Interestingly, the enhanced MUC16 binding with VRC01 was linked to higher G0 glycosylation whereas lower MUC16 binding and lower G0 glycosylation with RTX (Figure 3c). Similar results were observed with two additional HIV-specific mAbs, F240 and 2G12, where differential G0 content was linked to MUC16 binding (Supplementary Figure S4), further supporting a role for G0 glycosylation in preferential MUC16 binding, independent of antigen specificity.

To confirm the role of the G0 glycan in enhanced MUC16 binding, G0-Abs were enriched from HIVIG using the Erythina cristagalli lectin, which specifically binds to terminal $\beta 1$, 4-linked galactose, ${ }^{29}$ thus binding to G1/G2-containing Abs, and depleting these species, while enriching G0 structures in the unbound pool. G0-enriched Ab fractions bound MUC16 at approximately $80 \%$ of the amount of the input HIVIG, whereas G1/G2-enriched Ab fractions bound MUC16 at 20\% of input HIVIG (Figure 3d). Similarly, removal of the terminal galactose from HIVIG by enzymatic digestion with $\beta 1$, 4-galactosidase to generate $\mathrm{G} 0$-Abs resulted in increased binding to MUC16 compared with undigested Abs (Figure 3e), further supporting the hypothesis that G0-Abs have an enhanced capacity to bind to MUC16.

\section{Increased binding affinity to MUC16 is modulated by smaller Fc glycan structures}

To quantitatively measure the impact of the Fc glycan on binding to MUC16, we performed SPR analysis of the HIVspecific mAbs VRC01 and 2G12, which showed high and low MUC16 binding, respectively (Figure 2b, Supplementary Figure S4A), and polyclonal HIVIG after: (i) the enzymatic removal of sialic acid and galactose from the glycan, thus generating G0 glycoforms or (ii) after enzymatic removal of the 
a
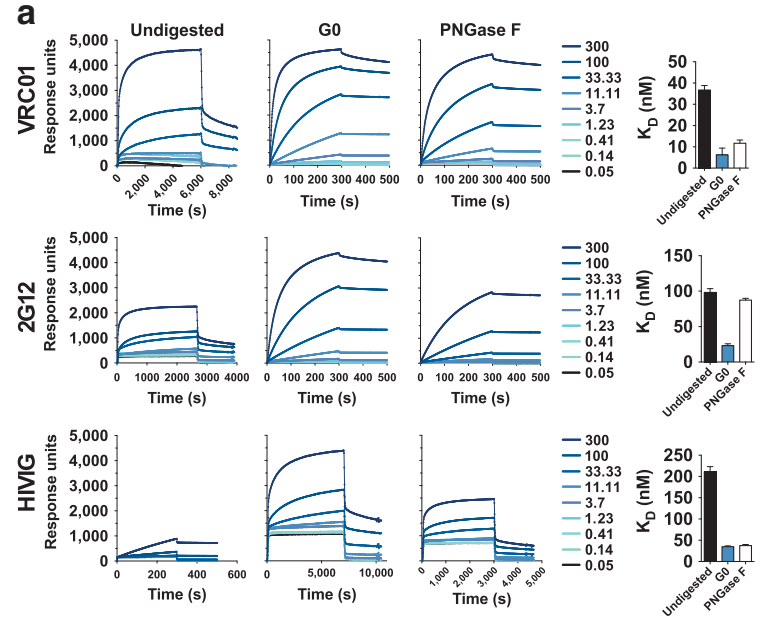
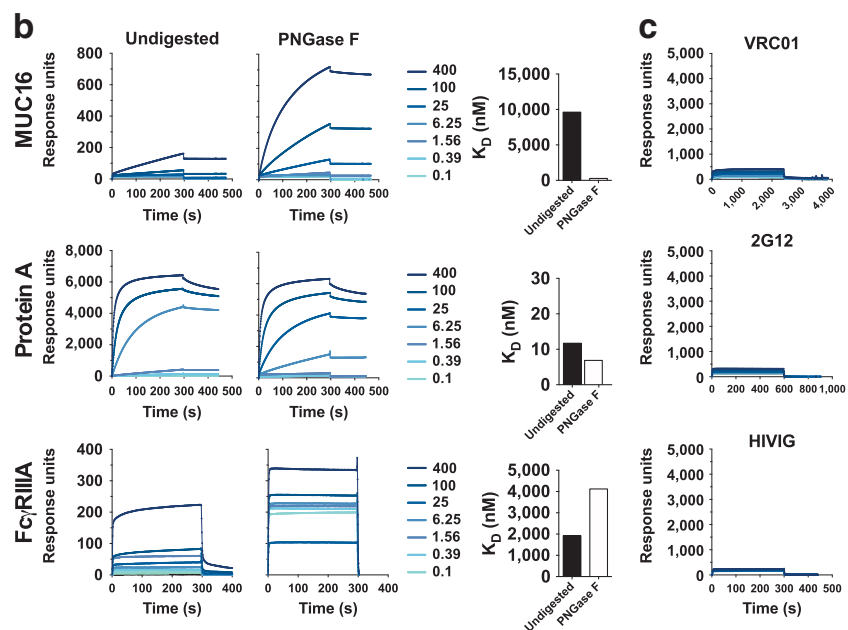

Figure 4 Increased binding affinity to MUC16 is modulated by smaller Fc glycan structures. (a) VRC01 (top), 2 G12 (middle), and HIVIG (bottom) were digested with enzymes to produce G0, or aglycosylated antibodies (Abs) and binding affinity to MUC16 was determined by surface plasmon resonance (SPR). Raw SPR curves and bar graphs of $K_{\mathrm{D}}$ values for indicated groups are shown. (b) Rituximab (RTX) was digested with PNGaseF to produce aglycosylated Abs, and binding affinity to MUC16 (top), protein A (middle) or Fc $\gamma$ RIIIA (bottom) was determined by SPR. Raw SPR curves and bar graphs of $K_{\mathrm{D}}$ values for indicated groups are shown. (c) N-glycans on MUC16 were removed by digestion with PNGaseF and binding affinity of indicated Abs to digested MUC16 was determined by SPR. Raw SPR curves are shown.

entire glycan by PNGaseF. Consistent with the ELISA data demonstrating that removal of galactose increases binding to MUC16 (Figure 3e), truncation of the glycan to G0 dramatically increased $\mathrm{Ab}$ affinity to MUC16 compared with undigested Abs (Figure 4a). Unexpectedly, the affinity of VRC01 and HIVIG binding to MUC16 was also increased when the Fc glycan was removed completely by PNGaseF (Figure 4a). Similarly, removal of the glycan increased the binding affinity of RTX to MUC16 compared with undigested $\mathrm{Ab}$ (Figure 4b). Conversely, RTX binding to Protein A, an Fc glycan-independent interaction, was not significantly altered with removal of the glycan, whereas binding to Fc $\gamma$ RIIIa, an Fc glycan-dependent interaction, was disrupted (Figure $\mathbf{4 b}$ ), as expected. $^{30,31}$ Of note, RTX does not have an N-linked glycosylation site in the Fab region, and only has an Fc glycan, thus the increased binding affinity following PNGaseF digestion supports the role of the Fc in mediating the interaction with MUC16 (Figure 2b,c). Together with the G0-MUC16 association within patient cohorts (Figure 3b, Supplementary Figure S2), these data provide compelling evidence that G0-containing Abs have greater affinity for MUC16. As G0 represents the smallest naturally occurring Fc glycan structure, these data suggest that smaller or no glycan structures confer enhanced MUC16 binding.

\section{MUC16 glycosylation is required for $\mathrm{Ab}$ binding}

Given the role of the Fc glycan in modulating Ab binding to MUC16, we hypothesized that the glycans on MUC16 may also modulate binding to the Abs. MUC16 is glycosylated with both O- and N-linked glycans that accounts for nearly $30 \%$ of the

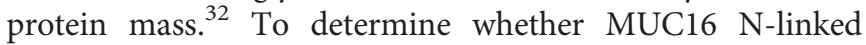
glycans modulate binding of Abs, MUC16 was digested with PNGaseF to remove $\mathrm{N}$-linked glycans, and the binding affinity to the mAbs was measured by SPR. Strikingly, PNGaseF treatment of MUC16 resulted in complete loss of binding to all Abs (Figure 4c), indicating that N-linked glycans on MUC16 are critical for $\mathrm{Ab}$ binding.

\section{Fucosylation also impacts MUC16 binding}

In addition to galactose, three additional sugars can be modified to alter $\mathrm{Ab}$ functionality: fucose reduces $\mathrm{Ab} \mathrm{Fc}$ binding to Fc $\gamma$ RIIIa, thus reducing ADCC; ${ }^{33}$ bisecting GlcNAc enhances binding to Fc $\gamma$ RIIIa thereby enhancing ADCC; ${ }^{34}$ and sialic acid can dampen inflammation and ADCC. ${ }^{35}$ To gain greater resolution of the glycan modifications that preferentially interact with MUC16, Abs from $\mathrm{HIV}^{+}$patients were incubated with beads coated with MUC16, and bound Abs were eluted before glycan characterization by mass spectrometry (Supplementary Figure S4). The frequency of specific Ab glycan structures in the total pool of Abs was compared with those in the MUC16-bound fraction to define the preferred structures that bound to MUC16. MUC16 pull-down captured the G0F glycan structure most abundantly, followed by G1F, G2F, G0FB, and G1FB (Figure 5a). Calculation of the percentage enrichment of MUC16-bound Abs compared with the input HIVIG demonstrated that MUC16-bound Abs were enriched for G0F, G1F, G2F, G1FB, and G0FB but reduced for G0B, G1B, and G2B (Figure 5b). Moreover, collective analysis of total G0, G1, and G2 glycans (including all substructures + / - fucose, sialic acid, and bisecting GlcNAc) demonstrated an enrichment of G0 structures in MUC16 bound Abs whereas G1 and G2 structures were reduced compared with input levels in $\mathrm{HIV}^{+}$ Abs (Figure 5c). Similarly, collective analysis of total glycan structures that include fucose and/or the bisecting GlcNAc highlights the preferential binding of all fucosylated Abs, but not afucosylated bisected glycosylated Abs (Figure 5d). 

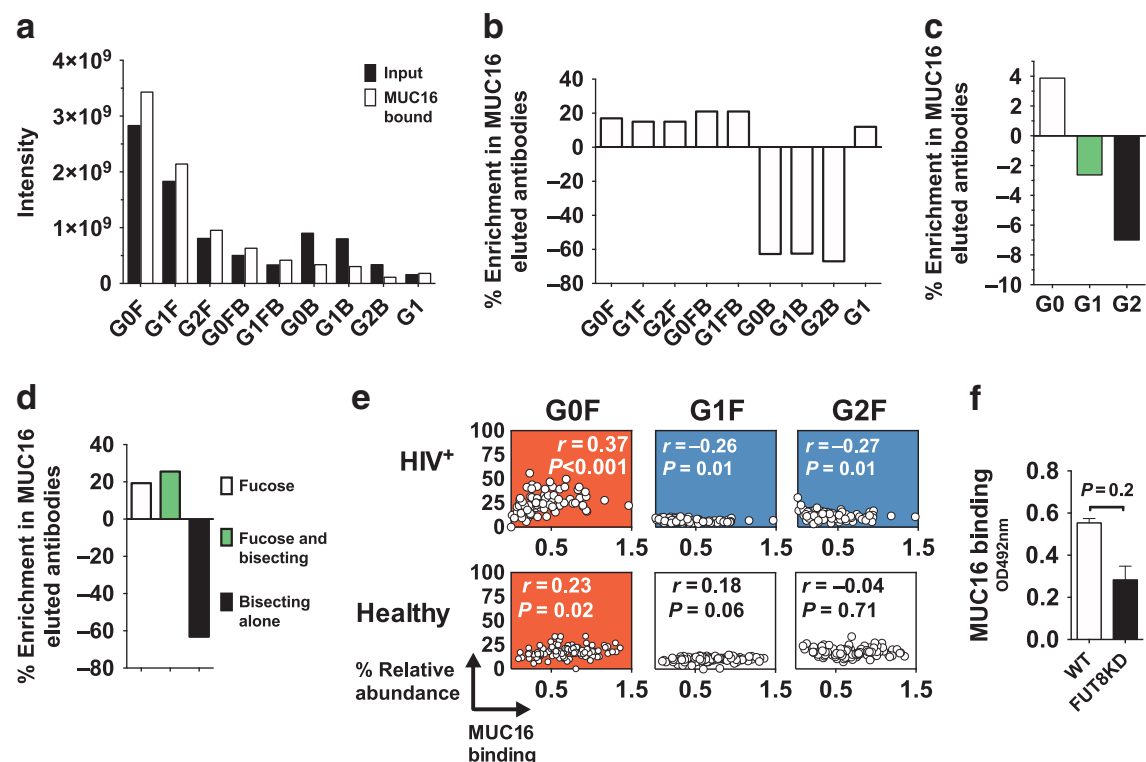

Figure 5 Agalactosylated and fucosylated antibodies (Abs) are enriched in mucin 16 (MUC16)-bound Abs. (a) MUC16-bound Abs were analyzed by mass spectrometry to determine glycan structure. The relative intensity values for input or MUC16-bound Abs for the indicated glycan are shown. (b) The percent enrichment of the indicated glycan structure in the MUC16-bound Ab pool compared with the input Ab pool is shown. (c, d) The percent enrichment of total G0, G1, or G2 glycan structures (c) or fucosylated (G0F, G1F, G2F), fucosylated and bisected (G0FB, G1FB) and bisected structures (G0B, G1B, G2B) (d) in the MUC16-bound Ab pool compared with the input Ab pool are shown. (e) The relative abundance of fucosylated glycan structures in bulk lgG from $\mathrm{HIV}^{+}$patients (top panel) or healthy subjects (bottom panel) was determined by capillary electrophoresis (CE). Spearman correlation coefficients indicate a significant positive (red), significant negative (blue), or no correlation (white) between relative glycan abundance and MUC16 binding. (f) VRC03 monoclonal Ab produced in either wild-type or FUT8kd 293T cells to produce fucosylated or afucosylated Abs, respectively, was assayed for MUC16 binding by ELISA.

Because fucose and the bisecting GlcNAc are typically (but not exclusively) added in a reciprocal order, ${ }^{36}$ reduced bisecting glycan binding may be related to a preferential interaction between MUC16 and fucosylated Abs. Moreover, the relative abundance of G0F-containing Abs correlated with MUC16 binding in both $\mathrm{HIV}^{+}$subject and healthy control populations (Figure 5e, Supplementary Figure S5), supporting the hypothesis that G0 fucosylated structures promote MUC16 binding. Finally, to test whether reducing fucosylation alters $\mathrm{Ab}$ binding to MUC16, the mAb VRC03 was produced either in WT or in stable FUT8 knockdown (the enzyme that adds fucose) 293T cells, the latter demonstrating reduced MUC16 binding (Figure 5f). Taken together, these data support a role for $\mathrm{Ab}$ fucosylation in promoting $\mathrm{Ab} / \mathrm{MUC16}$ interactions.

\section{MUC16-bound Abs captures HIV}

Capture of infectious HIV at mucosal barriers could serve to block transmission by immobilizing/neutralizing incoming HIV, leading to protective immunity. ${ }^{37,38}$ Given the apical localization of MUC16 above the epithelium (Figure 1c, Gipson $e \mathrm{al}^{8}$ ), MUC16-bound Abs may be well positioned to capture incoming virions at a distance from the underlying epithelium, thereby helping prevent infection. To determine whether MUC16-bound Abs could capture virus, fluorescent HIV viral particles were incubated with MUC16-bound HIVIG (10 or $100 \mu \mathrm{g} \mathrm{ml}^{-1}$ ) or Abs from a healthy subject $\left(100 \mu \mathrm{g} \mathrm{ml}^{-1}\right)$, and the number of HIV particles trapped by MUC16-bound Abs was quantified by confocal microscopy.
Although Abs from healthy controls trapped HIV particles to similar levels as MUC16 alone, MUC16-bound HIVIG trapped significantly more HIV particles at the highest concentration $\left(100 \mu \mathrm{g} \mathrm{ml}^{-1}\right)$ of HIVIG only, likely related to the low abundance of HIV-specific Abs within the polyclonal HIVIG pool (Figure 6a). ${ }^{39}$ In addition to the visualization of viral capture by microscopy, viral capture was quantified by p 24 ELISA. As G0 forms of Abs increase binding affinity to MUC16, the G0 glycoform of VRC01 was directly compared with the undigested VRC01. As shown in Figure 6b, VRC01 G0 captured higher amounts of HIV compared with undigested VRC01, and significantly higher virus than the healthy $\mathrm{Ab}$ control, indicating that increased MUC16 binding affinity results in increased viral capture. Together, these functional data indicate that MUC16-bound Abs not only bind to MUC16, but can capture HIV virions, potentially resulting in the sequestration of the virus above the epithelial border.

\section{DISCUSSION}

Reducing transmission of HIV across mucosal barriers is critical to ending the HIV epidemic, and identifying interactions that enhance mucosal protection will be a key to provide sterilizing immunity. Approaches aimed at naturally inducing $\mathrm{Ab}$ enrichment at mucosal barriers through vaccination or therapeutics could have a profound impact on limiting infection. Here, we demonstrate an interaction between the cell-associated mucin protein, MUC16, and Abs. Moreover, this interaction was preferentially enhanced in the context of 

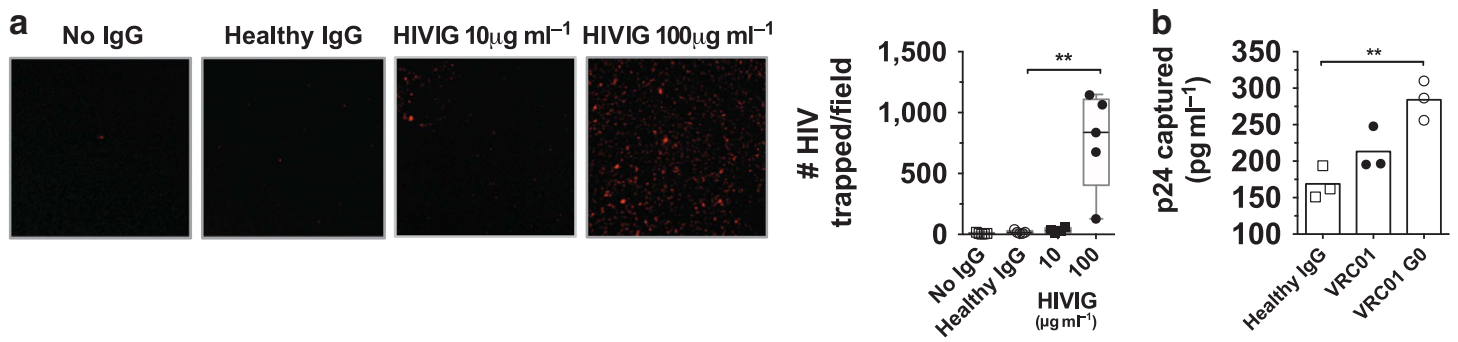

Figure 6 Mucin 16 (MUC16)-bound antibodies (Abs) capture HIV. (a) MUC16 was coated onto microscopy plates at $2 \mu \mathrm{g} \mathrm{ml}{ }^{-1}$. Abs were incubated at $10 \mathrm{~g} \mathrm{ml}^{-1}$ (HIVIG) or $100 \mu \mathrm{g} \mathrm{ml}^{-1}$ (HIVIG and healthy lgG) before washing and incubation with a fluorescent HIV (HIV-RFP). Trapped HIV was imaged by confocal microscopy (left), and the number of trapped virus was quantified using Image $\mathrm{J}$ (right). (b) Indicated Abs $\left(1 \mu \mathrm{g} \mathrm{ml}{ }^{-1}\right.$ ) were incubated with plate-bound MUC16, followed by incubated with $10 \mathrm{ng} \mathrm{ml}^{-1}$ of HIV (SF162). After $1 \mathrm{~h}$, plates were washed and the amount of virus captured was determined by p24 ELISA. ${ }^{* *} P<0.005$ by unpaired t-test.

chronic HIV infection, allowing us to identify a specific glycoform that confers enhanced binding and affinity to MUC16 and subsequent viral capture.

Although mucus alone slows the transit of viruses, the antiviral function of virus-specific Abs in mucus has been suggested in the context of herpes simplex virus-2 (HSV-2) where the presence of HSV-2-specific Abs within mucus decreases movement of that virus, correlating with reduced vaginal infection in mice. ${ }^{40}$ The synergy of Abs and mucus is likely mediated through multiple mechanisms including increasing the size of the pathogen by immune complex formation, and higher affinity interactions with particular mucus components such as mucins or additional Fc-interacting proteins. ${ }^{17,40,41}$ In the case of HSV-2, Ab glycosylation on HSV2 -specific Abs was critical in slowing virus transit through mucus, ${ }^{40}$ yet the mucus proteins engaged in this HSV-2 slowing process and specific $\mathrm{Ab}$ modifications that resulted in enhanced viral trapping are unclear. However, the results raised the exciting possibility that strategies aimed at increasing $\mathrm{Ab}$ abundance within mucus could effectively reduce pathogen infection across mucosal membranes. Here, we demonstrate a preferential interaction of Abs and MUC16 that can be enhanced by altering $\mathrm{Ab}$ glycosylation to shorter, agalactosylated fucosylated Abs that allows for capture of virus.

Generation of Abs with enhanced MUC16 binding capacity was not specific to HIV infection and was detectable in the setting of active RA (Figure 3a), but not in influenza or HCV infection, suggesting that specific inflammatory profiles, rather than infection per se, drive $\mathrm{Ab}$ modifications that enhance $\mathrm{Ab}$ binding to MUC16. Of note, while we only evaluated plasma Abs in this study, locally produced mucosal Abs may have distinct glycan profiles that allow for differential binding to mucosal proteins and will be probed in future studies. However, plasma Abs can access mucosal sites, ${ }^{42}$ allowing for enrichment of systemic Abs with enhanced MUC16 binding activity at MUC16-lined surfaces.

ELISA binding data, mass spectrometry analysis, and associations in patients point to the preferential binding of G0-containing Abs to MUC16 (Figures 3 and 5c). These data were validated by SPR studies, demonstrating that truncation of the Ab glycan to G0 on both polyclonal and monoclonal Abs dramatically increased the affinity of Ab binding to MUC16
(Figure 4a). Intriguingly, removal of the glycan by PNGaseF digestion also increased binding affinity of Abs to MUC16 (Figure 4a). The presence of different glycan structures (e.g., G0 vs. sialylated) or removal of glycan alters the $\mathrm{CH} 2$ domain, ${ }^{31,43-45}$ potentially impacting the flexibility/shape of the Ab, with G0 glycans representing the shortest naturally occurring structures, suggesting a possible mechanism of interaction between these two proteins. Alternatively, as the MUC16 N-linked glycans are critical for Ab binding (Figure 4c), it is possible that larger Fc glycans with multiple galactose and sialic acid residues may antagonize or hinder binding to MUC16 through glycan/glycan interactions, similar to the mechanism by which fucose hinders interaction with Fc $\gamma$ RIIIA. ${ }^{33}$ Future structural and glycosylation analyses may point to the specific mechanism of interaction, but together, our data demonstrate that Ab affinity to MUC16 can be tuned via alteration in galactosylation to potentiate viral capture.

In addition to the importance of G0 to improve MUC16 binding, natural Abs that preferentially bind to MUC16 tend toward enhanced fucosylation and reduced bisection. Both enhanced fucosylation ${ }^{33}$ and reduced bisection ${ }^{34}$ are independently linked to diminished ADCC activity, and thus the preference of fucosylated Abs by MUC16 may offer a noninflammatory advantage at mucosal membranes, as virusimmune complexes decorated with fucosylated Abs would limit highly-inflammatory cytolytic activity and pathology if detected by NK cells at mucosal membranes.

Methods to glyco-engineer Abs to produce particular glycan structures have been established, ${ }^{46}$ rendering it feasible to produce broadly neutralizing Abs with an enhanced capacity to bind to MUC16. We demonstrate here that HIV-specific mAbs can be modified to enhance MUC16 binding (Figure 4a), indicating that existing monoclonal therapeutics can be optimized to take advantage of enhanced MUC16 binding capacity to protect the underlying mucosa. Further, it is likely that particular vaccine vectors/adjuvants may selectively skew and tune Ab glycosylation to produce Abs with shorter glycans. Thus, next-generation vaccine design efforts may be able to modulate Ab/MUC16 interactions to trap virus within mucus, leading to enhanced protection from infection.

Beyond enrichment, enhancing $\mathrm{Ab}$ binding to MUC16 resulted in improved viral capture (Figure 6b). Viral capture 
has been associated with protection and reduced transmitted viruses, ${ }^{38,47}$ but $\mathrm{Ab}$ trapping of virus at the mucosa by nonneutralizing Abs may provide the virus with an enhanced capacity to infect. Although targeting neutralizing Abs to FcRn led to enhanced protection from infection, enrichment of nonneutralizing Abs on FcRn may result in more transcytosis of virus, and infection. ${ }^{48}$ MUC16 extends up to $300 \mathrm{~nm}$ into the lumen, forming a dense sheet of proteins that protect the underlying epithelium, ${ }^{49}$ thus virus trapped by MUC16-bound Abs will be sequestered above the epithelial surface and FcRn, and any capacity to transcytose. Moreover, MUC16 has a protease cleavage site that enables the ectodomain to be shed from the epithelial surface into mucus. ${ }^{49,50}$ Thus, it is plausible that upon immune complex mediated cross-linking of one or more MUC16 proteins, the complexes would be shed far from the surface in a larger complex with soluble MUC16 for removal in mucus.

MUC16 is present in multiple mucosal tissues, including the reproductive and respiratory tracts, thus approaches to enhance $\mathrm{Ab}$ binding to MUC16 offer a unique opportunity to coat mucosal surfaces to provide protection from additional viral, bacterial, and parasitic infections. Moreover, alternate $\mathrm{Ab}$ interactions likely exist for other mucin proteins, such as MUC5AC/MUC5B in the respiratory tract or MUC2 in the large intestine, ${ }^{51}$ offering additional opportunities and strategies to selectively program Abs to line specific mucosal barriers and improve anti-pathogen activity and protective efficacy. In summary, we describe a novel interaction between a mucosal protein, MUC16, and Abs that is enhanced through modulation of the Fc glycan, highlighting a novel opportunity to increase protection against virus transmission by enriching mucosal surfaces with HIV-specific Abs.

\section{METHODS}

Patient Abs and mAbs. Plasma samples from $\mathrm{HIV}^{+}, \mathrm{HCV}$, influenza, RA patients, and healthy subjects were obtained with MGH Institutional review board approval, and all patients provided written informed consent. Bulk IgG was purified and quantified as described in the Supplementary Methods. mAbs were purchased as indicated in the Supplementary Methods.

Binding ELISA. Recombinant galectins (R\&D Systems, Minneapolis, MN), MUC1 (aa1-264; Origene, Rockville, MD), MUC16 (aa2100521992; R\&D Systems), or native MUC16 isolated from OVCAR3 cells (see Supplementary Methods for details) were immobilized onto ELISA plates, and Abs were assayed for binding using detecting using $\alpha$-human IgG Fc (MP Cappel).

Microscopy. Cervical tissue was isolated from patients undergoing hysterectomies at Northwestern Memorial Hospital and explants of endocervix were preserved in OCT media (Study \#00025456). Tissue sections were stained as detailed in Supplementary Methods.

Cell-based Ab-MUC16 binding assay. WT or OVCAR3 cells stably expressing an shRNA targeting MUC16 were incubated with Cy5labeled Abs from chronic $\mathrm{HIV}^{+}$patients as detailed in Supplementary Methods. Binding of Abs to cells, defined by mean fluorescence intensity, was measured by flow cytometry.

Glycan analysis. The relative abundance of $\mathrm{Ab}$ glycan structures were quantified by capillary electrophoresis or mass spectrometry as previously described, ${ }^{27}$ detailed in Supplementary Methods.
Enzymatic digestions and glycan modification. Enzymatic digestion was used to modify glycans as described in Supplementary Methods. IgG was digested into Fab, $\mathrm{F}(\mathrm{ab})_{2}$, and $\mathrm{Fc}$ using IdeS (Genovis, Lund, Sweden) and papain (Thermo Scientific, Cambridge, MA). Glycans were digested with PNGaseF (NEB), neuraminidase (NEB), and $\beta 1,4$-galactosidase (EMD Millipore, Darmstadt, Germany). G0-Abs were enriched using ECL-agarose beads (Vectors Labs, Burlingame, CA) as described in Supplementary Methods.

SPR analysis. The binding affinity of digested Abs to recombinant MUC16 (R\&D), Fc $\gamma$ RIIIA (R\&D), and Protein A (Sigma-Aldrich, St. Louis, MO) was determined by SPR as detailed in Supplementary Methods.

MUC16 capture assay. MUC16 was bound to magnetic beads and incubated with pre-cleared HIVIG. Beads were washed, and bound Abs eluted in $6 \mathrm{M}$ guanidine $\mathrm{HCl}$ before analysis.

Afucosylated Ab production. 293T cells stably expressing FUT8 shRNA were generated and transfected with a plasmid expressing the heavy and light chains of IgG1 VRC03. Abs were purified by Protein A and evaluated for MUC16 binding as described above.

Viral capture assays. The amount of HIV capture by MUC16-bound Abs was determined by microscopy and p24 ELISA as detailed below and in Supplementary Methods.

Microscopy-based viral capture. MUC16 was bound to a polyd-lysine-coated microscopy plate (MatTek, Ashland, MA), and Abs were bound before the addition of fluorescent HIV ${ }^{52}$ Captured virions were imaged by confocal microscopy and enumerated using ImageJ.

ELISA-based viral capture. MUC16 was immobilized onto an ELISA plate, and Abs were bound before the addition of HIV SF162. Captured HIV was determined by p24 ELISA (Perkin-Elmer, Waltham, MA).

Statistical analysis. Data were analyzed for statistical significance using GraphPad Prism (San Diego, CA) as detailed in Supplementary Methods.

SUPPLEMENTARY MATERIAL is linked to the online version of the paper at http://www.nature.com/mi

\section{ACKNOWLEDGMENTS}

This work was supported by the Bill and Melinda Gates Foundation (BMGF) OPP1031734 (TJH). HIV + samples were collected under the BMGF OPP1066973 (B.D.W.). Ab glycan analysis was developed under the BMGF OPP1114729 (G.A.). HCV samples were collected under the NIH U19 A166345 (G.L.). Co-first authors contributed equally to this work including the identification of the Ab-MUC16 interaction (M.S.), performance of experiments (B.G./J.S./M.S.), and all figure and paper preparation (B.G./J.S.). We would like to thank Daniel Stieh for helpful advice pertaining to experimental design. We appreciate services and infrastructure support from Third Coast Center for AIDS Research (CFAR), an NIH funded center (P30 Al 117943) supported by NIAID, NHLBI, NIA, NIMHD, and NIMH.

\section{DISCLOSURE}

The authors declared no conflict of interest.

c) 2016 Society for Mucosal Immunology

\section{REFERENCES}

1. UNAIDS. Global Report: UNAIDS Report of the Global AIDS Epidemic 2013 (2013).

2. Shattock, R.J. \& Moore, J.P. Inhibiting sexual transmission of HIV-1 infection. Nat. Rev. Microbiol. 1, 25-34 (2003).

3. Hessell, A.J. et al. Effective, low-titer antibody protection against low-dose repeated mucosal SHIV challenge in macaques. Nat. Med. 15, 951-954 (2009).

4. Hessell, A.J. et al. Fc receptor but not complement binding is important in antibody protection against HIV. Nature 449, 101-104 (2007). 
5. Bournazos, S., Klein, F., Pietzsch, J., Seaman, M.S., Nussenzweig, M.C. \& Ravetch, J.V. Broadly neutralizing anti-HIV-1 antibodies require Fc effector functions for in vivo activity. Cell 158, 1243-1253 (2014).

6. Nag, P. et al. Women with cervicovaginal antibody-dependent cellmediated cytotoxicity have lower genital HIV-1 RNA loads. J. Infect. Dis. 190, 1970-1978 (2004).

7. Ko, S.Y. et al. Enhanced neonatal Fc receptor function improves protection against primate SHIV infection. Nature 2014).

8. Gipson, I.K. et al. MUC16 is lost from the uterodome (pinopode) surface of the receptive human endometrium: in vitro evidence that MUC16 is a barrier to trophoblast adherence. Biol. Reprod. 78, 134-142 (2008).

9. Blalock, T.D. et al. Functions of MUC16 in corneal epithelial cells. Invest. Ophthalmol. Vis. Sci. 48, 4509-4518 (2007).

10. Govindarajan, B. et al. A metalloproteinase secreted by Streptococcus pneumoniae removes membrane mucin MUC16 from the epithelial glycocalyx barrier. PloS One 7, e32418 (2012).

11. Hladik, F. \& McElrath, M.J. Setting the stage: host invasion by HIV. Nat. Rev. Immunol. 8, 447-457 (2008).

12. Raux, M. et al. IgG subclass distribution in serum and various mucosal fluids of HIV type 1-infected subjects. AIDS Res. Hum. Retroviruses 16, 583-594 (2000).

13. Dugast, A.S. et al. Independent evolution of FC- and Fab-mediated HIV-1specific antiviral antibody activity following acute infection. Eur. J. Immunol. 44, 2925-2937 (2014).

14. Argueso, P., Guzman-Aranguez, A., Mantelli, F., Cao, Z., Ricciuto, J. \& Panjwani, N. Association of cell surface mucins with galectin-3 contributes to the ocular surface epithelial barrier. J. Biol. Chem. 284, 23037-23045 (2009).

15. Kabawat, S.E., Bast, R.C. Jr., Bhan, A.K., Welch, W.R., Knapp, R.C. \& Colvin, R.B. Tissue distribution of a coelomic-epithelium-related antigen recognized by the monoclonal antibody OC125. Int. J. Gynecol. Pathol. 2, 275-285 (1983).

16. Kutteh, W.H., Hatch, K.D., Blackwell, R.E. \& Mestecky, J. Secretory immune system of the female reproductive tract: I. Immunoglobulin and secretory component-containing cells. Obstet. Gynecol. 71, 56-60 (1988).

17. Fahrbach, K.M., Malykhina, O., Stieh, D.J. \& Hope, T.J. Differential binding of $\lg G$ and $\lg A$ to mucus of the female reproductive tract. PloS One 8 , e76176 (2013).

18. Kui Wong, N. et al. Characterization of the oligosaccharides associated with the human ovarian tumor marker CA125. J. Biol. Chem. 278, 28619-28634 (2003).

19. Ackerman, M.E. et al. Natural variation in Fc glycosylation of HIV-specific antibodies impacts antiviral activity. J. Clin. Invest. 123, 2183-2192 (2013).

20. Tomaras, G.D. \& Haynes, B.F. HIV-1-specific antibody responses during acute and chronic HIV-1 infection. Curr. Opin. HIV AIDS 4, 373379 (2009)

21. Forthal, D., Hope, T.J. \& Alter, G. New paradigms for functional HIV-specific nonneutralizing antibodies. Curr. Opin. HIV AIDS 8, 393-401 (2013).

22. Arnold, J.N., Wormald, M.R., Sim, R.B., Rudd, P.M. \& Dwek, R.A. The impact of glycosylation on the biological function and structure of human immunoglobulins. Annu. Rev. Immunol. 25, 21-50 (2007).

23. Jefferis, R. Isotype and glycoform selection for antibody therapeutics. Arch. Biochem. Biophys. 526, 159-166 (2012).

24. Huhn, C., Selman, M.H., Ruhaak, L.R., Deelder, A.M. \& Wuhrer, M. IgG glycosylation analysis. Proteomics 9, 882-913 (2009).

25. Moore, J.S. et al. Increased levels of galactose-deficient lgG in sera of HIV1-infected individuals. AIDS 19, 381-389 (2005).

26. Parekh, R.B. et al. Association of rheumatoid arthritis and primary osteoarthritis with changes in the glycosylation pattern of total serum IgG. Nature 316, 452-457 (1985).

27. Mahan, A.E. et al. A method for high-throughput, sensitive analysis of lgG Fc and Fab glycosylation by capillary electrophoresis. J. Immunol. Methods 417, 34-44 (2015).

28. Frenzel, A., Hust, M. \& Schirrmann, T. Expression of recombinant antibodies. Front. Immunol. 4, 217 (2013).

29. Cummings, R.D. \& Etzler, M.E. Antibodies and lectins in glycan analysis. In: Essentials of Glycobiology, 2nd edn (Varki, A. et al., eds) (Cold Spring Harbor, NY, USA, 2009).
30. Deisenhofer, J. Crystallographic refinement and atomic models of a human Fc fragment and its complex with fragment B of protein A from Staphylococcus aureus at 2.9- and 2.8-A resolution. Biochemistry 20, 2361-2370 (1981).

31. Krapp, S., Mimura, Y., Jefferis, R., Huber, R. \& Sondermann, P. Structural analysis of human $\mathrm{lgG}-\mathrm{FC}_{\mathrm{C}}$ glycoforms reveals a correlation between glycosylation and structural integrity. J. Mol. Biol. 325, 979-989 (2003).

32. Yin, B.W. \& Lloyd, K.O. Molecular cloning of the CA125 ovarian cancer antigen: identification as a new mucin, MUC16. J. Biol. Chem. 276, 2737127375 (2001).

33. Ferrara, C. et al. Unique carbohydrate-carbohydrate interactions are required for high affinity binding between FcgammaRIII and antibodies lacking core fucose. Proc. Natl. Acad. Sci. USA 108, 12669-12674 (2011).

34. Davies, J., Jiang, L., Pan, L.Z., LaBarre, M.J., Anderson, D. \& Reff, M. Expression of GnTIII in a recombinant anti-CD2O CHO production cell line: expression of antibodies with altered glycoforms leads to an increase in ADCC through higher affinity for FC gamma RIII. Biotechnol. Bioeng. 74, 288-294 (2001).

35. Kaneko, Y., Nimmerjahn, F. \& Ravetch, J.V. Anti-inflammatory activity of immunoglobulin G resulting from Fc sialylation. Science 313, 670-673 (2006).

36. Longmore, G.D. \& Schachter, H. Product-identification and substratespecificity studies of the GDP-L-fucose:2-acetamido-2-deoxy-beta-Dglucoside (FUC goes to Asn-linked GlcNAc) 6-alpha-L-fucosyltransferase in a Golgi-rich fraction from porcine liver. Carbohydr. Res. 100, 365-392 (1982).

37. Liu, P. et al. Infectious virion capture by HIV-1 gp120-specific IgG from RV144 vaccinees. J. Virol. 87, 7828-7836 (2013).

38. Watkins, J.D. et al. Anti-HIV IgA isotypes: differential virion capture and inhibition of transcytosis are linked to prevention of mucosal R5 SHIV transmission. AIDS 27, F13-F20 (2013).

39. Morris, L. et al. HIV-1 antigen-specific and -nonspecific B cell responses are sensitive to combination antiretroviral therapy. J. Exp. Med. 188, 233-245 (1998).

40. Wang, Y.Y. et al. IgG in cervicovaginal mucus traps HSV and prevents vaginal Herpes infections. Mucosal Immunol. 7, 1036-1044 (2014).

41. Chen, A. et al. Transient antibody-mucin interactions produce a dynamic molecular shield against viral invasion. Biophys. J. 106, 2028-2036 (2014).

42. Russell, M.W. \& Mestecky, J. Humoral immune responses to microbial infections in the genital tract. Microbes Infect. 4, 667-677 (2002).

43. Buck, P.M., Kumar, S. \& Singh, S.K. Consequences of glycan truncation on Fc structural integrity. mAbs 5, 904-916 (2013).

44. Mimura, Y. et al. The influence of glycosylation on the thermal stability and effector function expression of human IgG1-Fc: properties of a series of truncated glycoforms. Mol. Immunol. 37, 697-706 (2000).

45. Hanson, Q.M. \& Barb, A.W. A perspective on the structure and receptor binding properties of immunoglobulin g fc. Biochemistry 54, 2931-2942 (2015).

46. Dalziel, M., Crispin, M., Scanlan, C.N., Zitzmann, N. \& Dwek, R.A. Emerging principles for the therapeutic exploitation of glycosylation. Science 343, 1235681 (2014).

47. Santra, S. et al. Human non-neutralizing HIV-1 envelope monoclonal antibodies limit the number of founder viruses during SHIV mucosal infection in Rhesus Macaques. PLoS Pathog. 11, e1005042 (2015).

48. Gupta, S. et al. The Neonatal Fc receptor ( $\mathrm{FCRn})$ enhances human immunodeficiency virus type 1 (HIV-1) transcytosis across epithelial cells. PLoS Pathog. 9, e1003776 (2013).

49. Hattrup, C.L. \& Gendler, S.J. Structure and function of the cell surface (tethered) mucins. Annu. Rev. Physiol. 70, 431-457 (2008).

50. Blalock, T.D., Spurr-Michaud, S.J., Tisdale, A.S. \& Gipson, I.K. Release of membrane-associated mucins from ocular surface epithelia. Invest. Ophthalmol. Vis. Sci. 49, 1864-1871 (2008).

51. McGuckin, M.A., Linden, S.K., Sutton, P. \& Florin, T.H. Mucin dynamics and enteric pathogens. Nat. Rev. Microbiol. 9, 265-278 (2011).

52. Chukkapalli, V., Hogue, I.B., Boyko, V., Hu, W.S. \& Ono, A. Interaction between the human immunodeficiency virus type 1 Gag matrix domain and phosphatidylinositol-(4,5)-bisphosphate is essential for efficient gag membrane binding. J. Virol. 82, 2405-2417 (2008). 